\title{
Hernia umbilical: un problema no resuelto
}

\author{
Alejandro Lora-Aguirre', Neil Valentín Vega-Peña'-2, Arnold José Barrios-Parra', Juan Pablo Ruiz-Pineda'
}

\section{RESUMEN}

La cirugía de la hernia umbilical ha sido considerada tradicionalmente como sencilla y de fácil ejecución. El cambio conceptual de la hernia umbilical y los avances en su tratamiento han modificado su abordaje, estableciéndose escenarios de complejidad variable (obesidad, embarazo, cirróticos, distasis de los rectos, etc.) que demandan un conocimiento más profundo del tema por parte de la comunidad médica. El impacto económico en el sistema de salud, debido a su alta prevalencia como patología quirúrgica, implica un uso racional de recursos, así como la necesidad de una nueva categorización dentro de la cirugía de la pared abdominal. Es necesario establecer modificaciones en los procesos diagnósticos y terapéuticos en una entidad que ha sido relegada a los niveles básicos del ejercicio quirúrgico habitual.

\section{PALABRAS CLAVE}

Cirugía; Diagnóstico; Hernia Umbilical; Medicina Basada en Evidencia; Prótesis

\section{SUMMARY}

\section{Umbilical hernia: an unsolved problem}

Umbilical hernia surgery has traditionally been considered simple and easy to perform. A conceptual change of the umbilical hernia and the advances in its treatment have modified its approach, establishing scenarios of variable complexity (Obesity, pregnancy, cirrhosis, diastasis of the rectus, etc.), which demand a deeper knowledge of the subject on the medical community. The economic impact on the health system, due to its high prevalence as a surgical pathology, implies a

\footnotetext{
1 MD, Departamento de Cirugía, Clínicas Colsanitas, Fundación Universitaria Sanitas, Bogotá, Colombia. Grupo de Cirugía de la Pared Abdominal.

2 MD, MSC, FACS; Profesor Asistente de Cirugía, Universidad de La Sabana, Chía, Colombia.
}

Correspondencia: Neil Valentín Vega-Peña; neil.valentin@unisabana.edu.co

Recibido: enero 23 de 2019

Aceptado: abril 29 de 2019

Cómo citar: Lora-Aguirre A, Vega-Peña NV, Barrios-Parra AJ, Ruiz-Pineda JP. Hernia umbilical: un problema no resuelto. latreia. 2019 0ct-Dic;32(4):288-297. D0I 10.17533/udea.iatreia.28. 
rational use of resources, as well as the need for a new categorization within the abdominal wall surgery. It is necessary to establish modifications in the diagnostic and therapeutic processes in an entity that has been relegated to the basic levels of the usual surgical practice.

\section{KEYWORDS}

Diagnosis; Evidence-Based Medicine; Hernia, Umbilical; Surgery; Prosthesis

\section{INTRODUCCIÓN}

The humble umbilical hernia remains a simple yet complex problem. It's final chapter remains to be written ${ }^{(1)}$.

La hernia umbilical se considera, de acuerdo a la clasificación de los defectos de la pared abdominal de la Asociación Europea de la Hernia, como una hernia primaria de la línea media dentro de los márónenes laterales de las vainas de los músculos rectos, en un rango de $3 \mathrm{~cm}$ por encima y por debajo del ombligo (1). Su conceptualización ha evolucionado desde una "hernia simple" hacia una entidad diferente, dados los avances en su diagnóstico y tratamiento ${ }^{(2.3)}$.

La presente revisión pretende actualizar al lector en los temas controversiales e innovadores respecto al tratamiento de la hernia umbilical, con el esperado impacto favorable en los desenlaces quirúrgicos.

\section{GENERALIDADES}

Las hernias umbilicales constituyen entre el 6-14\% de las hernias de la pared abdominal en adultos, de las cuales el $90 \%$ son adquiridas, con predominio en mujeres, en una relación de 5:1 ${ }^{(3-4)}$; son comunes en la infancia y alrededor del $80 \%$ se obliteran entre los 4 y 5 años ${ }^{(5)}$.

El embarazo, la obesidad, los tumores, la ascitis y actividad física, entre otros, aumentan la presión intrabdominal, haciendo manifiesta su presencia al incrementar el diámetro de los anillos umbilicales, usualmente imperceptibles ${ }^{(4,6)}$.

\section{CLÍNICA Y DIAGNÓSTICO}

La presentación oscila desde asintomática hasta una condición catastrófica con encarcelamiento, necrosis de la piel y del contenido herniario. Esta variabilidad influye en la percepción y priorización del tratamiento quirúroico, especialmente en un escenario no urgente ${ }^{(3)}$.

Los estudios imagenológicos son útiles en los pacientes obesos (defectos asociados de la pared), hernias gigantes (contenido y anatomía de la hernia), antecedentes quirúrgicos de la pared abdominal, ante cambios en las características de las hernias inveteradas o ante un dolor umbilical sin una evidente manifestación clínica de hernia ${ }^{(6,7)}$ (Figura 1).

\section{EVALUACIÓN PREOPERATORIA}

Incluye una valoración del dolor y su relación con la hernia, síntomas gastrointestinales asociados, la limitación funcional y las expectativas del paciente, los tratamientos a realizar, así como las enfermedades y antecedentes asociados. Cabe anotar que los pacientes con pequeñas hernias asintomáticas en quienes haya cambiado la condición a sintomáticos, o quienes refieran un aumento del tamaño de su hernia, pueden tener un aumento de la presión intrabdominal que debe ser estudiado, constituyéndose en estos casos la hernia umbilical en un siono clínico de una condición intrabdominal de mayor complejidad.

Clínicamente deben evaluarse el tamaño del contenido herniario, la relación del saco del anillo herniario, la supuración umbilical y las condiciones propias de cada paciente, estratificando el riesgo de recidiva y los eventos del sitio operatorio. Según lo anterior, se definirá la pertinencia de las imágenes diagnósticas complementarias ${ }^{(7)}$.

\section{TRATAMIENTO QUIRÚRGICO}

La variabilidad de las presentaciones clínicas y las técnicas quirúrgicas empleadas dificultan unificar los criterios en el tratamiento, considerándose un enfoque quirúrogico específico para cada paciente con hernia, con "Ia mejor opción" según sea el caso. A continuación se relacionan los diferentes abordajes quirúrogicos disponibles. 


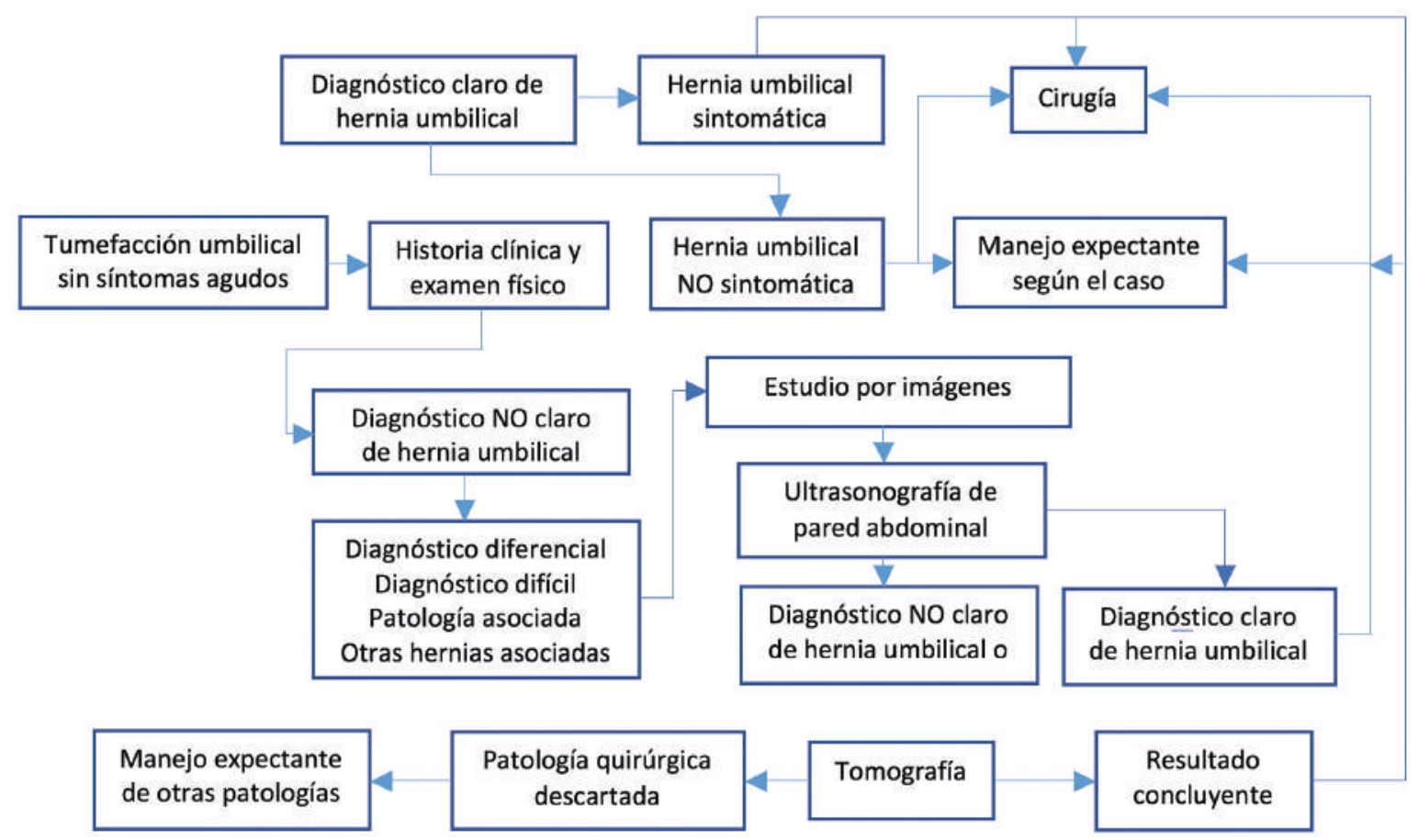

Figura 1. Flujograma diagnóstico. Pacientes con sospecha de hernia umbilical. Fuente: modificada de la referencia ${ }^{(3)}$

\section{Reparaciones con malla o sin malla}

La utilización rutinaria de las prótesis tiene un impacto favorable en las recidivas, aún a pesar de mayores eventos adversos del sitio operatorio (seroma, infección o hematoma, entre otros) y el dolor crónico resultantes ${ }^{(8,9)}$, documentándose durante los últimos 15 años una reducción de las recidivas, desde $11,5 \%$ hasta $0 \%{ }^{(10)}$, de $9,8 \%$ hasta $2,4 \%{ }^{(1)} y$ de $8,2 \%$ hasta 2,7 $\%{ }^{(8)}$, según varios estudios analíticos.

Un ensayo clínico multicéntrico efectuado en 300 pacientes, evidencia el beneficio de la utilización de prótesis en defectos de 1 a $4 \mathrm{~cm}$ de diámetro, al disminuir la tasa de recidiva herniaria a 3,6\% frente a un $11,4 \%$ en el cierre primario ( $-\mathrm{HR}=0,31$; IC $95 \%$ $=0,12-0,80$ - NNT 13 pacientes), tras un seguimiento promedio de 25 meses ${ }^{(12)}$.

La evaluación de la evidencia mediante una revisión sistemática y metanálisis reciente, que incluyó cinco ensayos clínicos controlados, concluyó que la utilización de una prótesis en la reparación de la hernia umbilical protege significativamente contra la recurrencia, comparada con las reparaciones no protésicas, en defectos mayores de un centímetro de diámetro $\left(\mathrm{RR}=0,28 ;\right.$ IC $95 \%=0.13-0,58-\mathrm{I}^{2}=0 \%-\mathrm{NNT}=$ 13 pacientes), sin diferencias en el riesgo de infección $(\mathrm{RR}=0,80 ; \mathrm{IC} 95 \%=0,36-1,79)$, seroma $(\mathrm{RR}=1,38$; IC $95 \%=0,57-3,32)$ o hematoma $(R R=0,55$; IC $95 \%$ $=0,23-1,30)^{(13)}$.

A pesar de lo anterior, el uso rutinario de las prótesis no se considera un estándar del manejo por la mayoría de los cirujanos, quienes son tradicionales y se resisten al cambio ${ }^{(14)}$. Ver las recomendaciones para la utilización de las prótesis (Tabla 1).

\section{Reparo abierto sin malla}

Es la técnica utilizada con mayor frecuencia, a pesar de una alta recidiva ${ }^{(13)}$, con diferentes materiales de sutura y formas de efectuar la reparación herniaria ${ }^{(3)}$.

Ventajas y desventajas teóricas de las diferentes opciones y materiales en el cierre primario (Tabla 2). 
Tabla 1. Consideraciones para el uso de prótesis en hernia umbilical

\begin{tabular}{cc}
\hline Favorece el uso de prótesis & Favorece el cierre primario \\
Defectos mayores a $1 \mathrm{~cm}$ & $\begin{array}{c}\text { Defectos menores a } 1 \mathrm{~cm} \text {, sin factores de riesgo } \\
\text { Infección activa de la piel: ulceración }\end{array}$ \\
$\begin{array}{c}\text { Condiciones de presión intrabdominal elevada } \\
\text { Hernia umbilical recidivada }\end{array}$ & $\begin{array}{c}\text { Estrangulación herniaria } \\
\text { Cicatrización anormal: }\end{array}$ \\
uso de esteroides, inmunosupresores, ascitis & No hay disponibilidad de prótesis adecuada \\
Trastornos del colágeno: herniosis. & Por elección informada del paciente: cualquier razón \\
Diástasis de los rectos asociada &
\end{tabular}

Fuente: referencias $(3,8,9)$

Tabla 2. Consideraciones sobre los materiales y las técnicas del cierre primario

\begin{tabular}{|c|c|c|c|}
\hline \multicolumn{4}{|c|}{ Consideraciones del reparo primario: selección de la sutura } \\
\hline Material de sutura & \multirow{2}{*}{ Ventajas } & \multirow{2}{*}{ Desventajas } & \multirow{2}{*}{ Comentarios } \\
\hline Técnica quirúrgica & & & \\
\hline No absorbible & $\begin{array}{l}\text { Mínima pérdida de la fuerza tensil } \\
\text { de la sutura. }\end{array}$ & $\begin{array}{l}\text { Incrementa el riesgo a largo pla- } \\
\text { zo de granulomas, dolor crónico } \\
\text { y sensación de cuerpo extraño. }\end{array}$ & $\begin{array}{l}\text { Riesgo mayor con suturas trenza- } \\
\text { das multifilamentosas. }\end{array}$ \\
\hline $\begin{array}{l}\text { Lentamente } \\
\text { absorbible }\end{array}$ & $\begin{array}{l}\text { Alta fuerza tensil durante el período } \\
\text { de cicatrización necesario (6 a } 12 \\
\text { semanas) Desaparición de la sutura. }\end{array}$ & $\begin{array}{l}\text { Mayor memoria inelástica que } \\
\text { dificulta su manejo. }\end{array}$ & $\begin{array}{l}\text { Las suturas barbadas monofila- } \\
\text { mento están disponibles. Menor } \\
\text { formación de granulomas. }\end{array}$ \\
\hline $\begin{array}{l}\text { Cierre de una } \\
\text { sola capa }\end{array}$ & Técnicamente fácil. & $\begin{array}{l}\text { No hay redundancia de los } \\
\text { tejidos a unir. }\end{array}$ & Técnica más empleada. \\
\hline $\begin{array}{l}\text { Cierre multicapa } \\
\text { (imbricación) }\end{array}$ & $\begin{array}{l}\text { Distribuye la tensión sobre } 2 \text { capas, } \\
\text { creando redundancia. }\end{array}$ & $\begin{array}{l}\text { Más demandante. } \\
\text { Mayor tiempo quirúrgico. } \\
\text { Aumento de las recidivas. }\end{array}$ & $\begin{array}{c}\text { Debe tener orientación transversa. } \\
\text { Precaución en los puntos profun- } \\
\text { dos en la capa final } \\
\text { (Lesión visceral). }\end{array}$ \\
\hline
\end{tabular}

Fuente: elaboración propia (experiencia de los autores)

\section{Reparo abierto con malla}

Implica crear un espacio anatómico o modificar uno existente con un esfuerzo técnico para ello. También parecen lograrse con los materiales protésicos de monofilamento de densidad intermedia $(40-80 \mathrm{gr} / \mathrm{m} 2)$ y macro porosos (poros de $1-3 \mathrm{~mm})^{(13,14)}$ una mejoría en la integración, resistencia a la infección, formación de granulomas y menor sensación de cuerpo extraño o dolor crónico, pudiendo efectuarse en los siguientes sitios:

- Detrás de los músculos rectos del abdomen (preperitoneal o sublay): es difícil en obesos y en reproducciones herniarias.

- Intermuscular sin cerrar el defecto (inlay): tiene alta recidiva, no recomendable.
- Sobre la aponeurosis (onIay): de fácil instalación, con mayor riesgo de granulomas y seromas ${ }^{(6,15)}$.

Se puede realizar una fijación no invasiva de las prótesis mediante la utilización de fibrina o pegantes sintéticos, o también una fijación invasiva de la forma tradicional con sutura, recomendándose un solapamiento mínimo de $3 \mathrm{~cm}$ con relación a los bordes del defecto herniario, según el manejo de las hernias ventrales ${ }^{(16,17)}$.

Las prótesis del tipo parche requieren una barrera antiadherente que aumenta su costo, debido a su posición intraperitoneal, sin diferencias en recidivas, infección y dolor postoperatorio al compararse con las prótesis planas tradicionales ${ }^{(18,19)}$. 


\section{Reparación mínimamente invasiva}

Se considera en las hernias encarceladas, agudas o crónicas, diástasis de los rectos asociados, sospecha de otras hernias concomitantes u ocultas, defectos mayores de $4 \mathrm{~cm}$, obesidad o recidivas e, incluye el uso de una prótesis con o sin el cierre del defecto herniario ${ }^{(20,21)}$.

La técnica más empleada es el IPOM (malla intraperitoneal) con prótesis recubiertas y dispositivos de fijación a la pared abdominal. Sus ventajas derivan de las menores tasas de eventos del sitio operatorio, tiempos quirúrgicos y estancia hospitalaria en casos complejos, con limitaciones dadas por la logística necesaria, los costos totales del procedimiento, la severidad de las complicaciones (lesiones vasculares e intestinales) y la curva de aprendizaje del cirujano ${ }^{(20)}$. La recidiva, el dolor y la calidad de vida son comparables con la cirugía abierta ${ }^{(20,22,23) .}$

Con los avances en la cirugía mínimamente invasiva se ha popularizado la instalación de mallas no recubiertas en el espacio preperitoneal y retro muscular, disminuyendo los riesgos de un cuerpo extraño en la cavidad peritoneal (IPOM) y los costos totales del procedimiento, a expensas de aumentar la complejidad de este y la logística requerida para su ejecución ${ }^{(24)}$.

Aunque la cirugía robótica en el campo de la hernia ha ido en aumento, no es fácilmente aceptada por los cirujanos y las instituciones de acuerdo con los costos y la utilidad clínica cuestionada en casos no complejos, lo cual se potencia en la hernia umbilical ${ }^{(25)}$.

\section{HERNIAS EN PACIENTES ASINTOMÁTICOS: ¿ESPERAR Y OBSERVAR?}

En pacientes asintomáticos la observación constituye una alternativa viable, teniendo en cuenta la alta probabilidad de recurrencia en reparaciones no protésicas, comparada con las eventuales complicaciones de una conducta expectante no quirúroica $(3,26)$.

La posibilidad de predecir complicaciónes en una hernia asintomática fue evaluada mediante el cálculo de la relación entre el saco herniario (SHU) y el aniIlo herniario umbilical (AHU) con ecografía o tomografía abdominal donde valores superiores a 2,5 de esta relación saco/hernia, incrementan el riesgo de complicaciones (índice SHU/AHU > 2,5 - OR 53,24; $95 \%$ CI: $12,77-345,20)$ con alto poder predictor de las mismas (AUC 0,90 - $91 \%$ sensibilidad y $84 \%$ especificidad) además se describe su utilidad en la toma de decisiones al ofrecer una conducta expectante ${ }^{(27)}$. A pesar del potencial beneficio del trabajo, sus limitaciones están dadas por su carácter de evaluación retrospectiva que debe ser validada en contextos diferentes y de forma prospectiva.

El manejo expectante en pacientes asintomáticos (watchful waiting) se ha efectuado de forma limitada con abordajes basados en desenlaces clínicos y económicos:

- Una evaluación retrospectiva establece una probabilidad de ciruogía del 4,3 \% al cabo de dos años, motivada por emergencia o dolor, sin alteraciones en la calidad de vida en los no intervenidos, aunque con limitaciones en los tamaños de muestra y pérdidas del seguimiento ${ }^{(26)}$.

- Una búsqueda de posibles factores condicionantes de resultados quirúrogicos ideales (ausencia de síntomas y baja recurrencia) en hernias umbilicales grandes encontró que los mismos son "sorprendentemente bajos" a pesar de la técnica y el uso de prótesis, resultando en dolor y limitación para la actividad diaria. Los autores proponen cirugía temprana en todos los defectos menores de $9 \mathrm{~cm}^{2}$ e incluso en hernias asintomáticas ${ }^{(28)}$.

- La medición de los costos en una estrategia expectante encontró un aumento en los mismos en los pacientes operados (atribuidos a la cirugía por sí misma), pero menores a largo plazo tras un año de seguimiento. Los autores contemplan una mayor utilización de recursos del sistema de salud e incapacidades en los pacientes asintomáticos, apoyando un tratamiento quirúrgico precoz ${ }^{(29)}$. Estos resultados deben ser interpretados con cuidado debido al contexto regional, la población estudiada y la metodología empleada: estudio observacional retrospectivo en EE.UU. con aseguramiento privado y patrocinado por la industria farmacéutica ${ }^{(29)}$.

Con base en lo anterior podemos afirmar que son candidatos para la observación de los defectos umbilicales pequeños asintomáticos los pacientes con comorbilidades no compensadas, pacientes menores de dos años de edad ${ }^{(5)}$, posiblemente, aquellos con un índice hernia/saco $<2,5$ y en quienes no acepten la opción quirúrogica. 


\section{HERNIA UMBILICAL EN LOS PACIENTES CON CIRROSIS}

Su prevalencia asciende hasta un $20 \%, 10$ veces mayor que la población general, producto de la combinación de ascitis, desnutrición y debilidad de la pared abdominal anterior, condiciones propias de estos pacientes ${ }^{(30)}$. Existe un aumento de la presion intraabdominal que ocasiona eversión y ampliación del orificio umbilical, ubica al peritoneo en contacto con la piel, ulcerándose la misma y generando una fistula peritoneo-cutanea con drenaje de ascitis al exterior del abdomen ${ }^{(30)}$.

La ascitis y la función hepática deben evaluarse con las escalas pronósticas propias de los pacientes en espera de trasplante hepático como la escala de CHILD
-POUG o el MELD ("Model for end-stage liver disease"), con los respectivos valores críticos para complicaciones y mortalidad, determinados por puntajes de CHILD B o superiores y, niveles MELD mayores a 10 puntos, requiriéndose una optimización preoperatoria en estos pacientes ${ }^{(31,32)}$.

La mortalidad calculada varía según el escenario, siendo mayor en casos de cirugía de urgencia con cifras de $6-8 \%$ vs. $2-3 \%$ en cirugía programada ${ }^{(31)} ;$ y depende, entre otros factores, del estado de la hernia, la presencia de infección local o sistémica, así como de la magnitud del procedimiento ${ }^{(3,32)}$. La cirugía electiva está supeditada al control de la ascitis, incluyendo la utilización de TIPS (derivación portosistémica intrahepática transyugular) o drenajes repetidos (Figura 2).

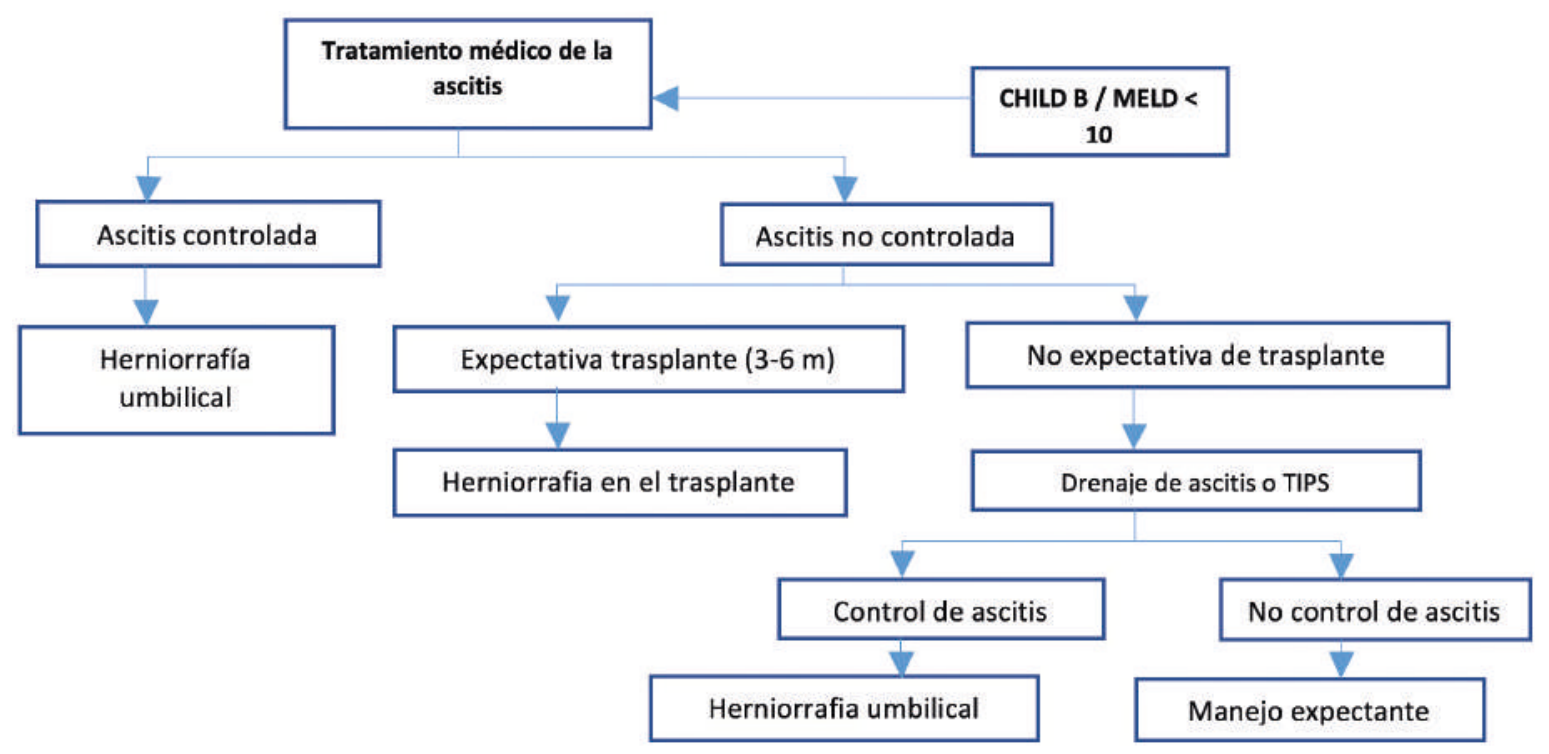

Figura 2. Manejo de la hernia umbilical en los pacientes con cirrosis Modificada de Coelho JCU, et al., ${ }^{(32)}$

De acuerdo con lo anteriormente expuesto, se requiere una evaluación del riesgo-beneficio del procedimiento. EI manejo expectante se asocia con una tasa elevada de complicaciones y morbimortalidad como encarcelamiento, evisceración, fístula peritoneocutánea y drenaje de ascitis, entre otros. Por el contrario, la cirugía electiva en el paciente con cirrosis compensada puede ser realizada con menores complicaciones, siendo la recomendación actual de manejo. EI control de la ascitis debe procurarse en todos los pacientes en relación directa con la compensación de su estado de hipertensión portal, incluso mediante la utilización de TIPS preoperatoriamente, con adecuado control de la infección y la coagulación, 
redundando en una optimización del paciente sin desconocer su condición de riesgo quirúrgico superior al paciente sin cirrosis ${ }^{(31,32)}$.

\section{HERNIA UMBILICAL Y CONDICIÓN DE EMBARAZO}

El manejo depende de los síntomas y el estado deI embarazo al momento del diagnóstico. Se debe considerar el eventual impacto en el binomio madre-hijo, así como el aumento transitorio y fisiológico de la presión intrabdominal. Podría resumirse de la siguiente manera ${ }^{(33)}$ :

Hernia pequeña, mínimos síntomas o asintomática: diferir manejo al término del embarazo.

Hernia grande, progresiva o incapacitante: reparación electiva en el segundo semestre $\nabla s$. reposo y diferir después del parto.

Hernia complicada (encarcelada o estrangulada): cirugía de emerogencia e independiente del trimestre del embarazo.
La cirugía durante el embarazo es infrecuente. En escenarios de urgencia tiene bajas complicaciones para la madre o el embarazo ${ }^{(34)}$, prefiriéndose en el segundo trimestre de la gestación y bajo condiciones de emergencia que justifiquen asumir dicho riesgo.

La herniorrafia umbilical concomitantemente con la cesárea es una práctica aceptada, genera satisfacción en las pacientes, debe discutirse en casos sintomáticos y de progresión herniaria, evaluando los pros y contras de la utilización de materiales protésicos en este contexto ${ }^{(35)}$ (Tabla 3).

Debido al carácter transitorio de los cambios hormonales $y$ de los músculos de la pared abdominal durante la gestación (estudios de electromiografía documentan retorno de la actividad de los músculos de la pared abdominal a las 18 semanas posparto), parece prudente un año como intervalo para una cirugía, esperando la recuperación del peso normal, la estabilización hormonal y el crecimiento del recién nacido ${ }^{(33,36)}$.

Tabla 3. Consideraciones de la utilización de prótesis y las condiciones de la gestación en las pacientes con hernia umbilical

\begin{tabular}{ccc}
\hline Condicion clínica & Reparo primario & Reparacion protésica \\
Expectativa de nuevo embarazo & Riesgo mayor de recurrencia & Posibilidad de dolor en el tercer trimestre \\
Diagnóstico en el embarazo & Riesgo mayor de recurrencia & Riesgo mayor de infección \\
Parto por cesárea & $\begin{array}{c}\text { Cirugía simultánea sin incisión separada, } \\
\text { riesgo mayor de recurrencia }\end{array}$ & $\begin{array}{c}\text { Incisión separada, } \\
\text { riesgo mayor de infección }\end{array}$ \\
Cirugía posparto & Tiempo óptimo individualizado & Tiempo óptimo individualizado \\
Diástasis de los rectos & Riesgo mayor de recurrencia & $\begin{array}{c}\text { Recomendada, } \\
\text { opción discutida con la paciente }\end{array}$ \\
\hline
\end{tabular}

Fuente: referencias $(33,35)$

\section{HERNIA UMBILICAL Y PACIENTES CON DIÁSTASIS DE LOS RECTOS ABDOMINALES}

La diástasis de los rectos tiene una prevalencia estimada de un 30-70 \% en la población general, condicionando una zona de debilidad en la pared abdominal anterior ${ }^{(37)}$. No se considera una verdadera hernia y se ha conceptualizado de forma hipotética como resultante del complejo de la Línea Alba enferma
(SCLA), proponiendo su resección para un adecuado cierre de la pared abdominal ${ }^{(38)}$.

De acuerdo con esta teoría, cualquier sutura efectuada en dicho complejo tiene una alta probabilidad de fracasar independiente del material o la técnica quirúrogica, de acuerdo a las alteraciones morfológicas y estructurales del tejido $y$, por consiguiente, la reparación de una hernia umbilical sintomática asociada a esta condición implica la corrección concomitante 
de la diástasis de los rectos, con o sin la instalación de una malla que genere un refuerzo en la pared abdominal anterior ${ }^{(39)}$. Esto último puede realizarse con cirugía abierta o mínimamente invasiva, en los espacios onlay, sublay, retromuscular o IPOM, de acuerdo a las preferencias, competencias del cirujano y los recursos institucionales disponibles ${ }^{(37)}$.

El cierre de una diástasis de los rectos abdominales sin utilizar una prótesis es una opción de tratamiento a considerar, aunque no hay estudios comparativos aleatorizados que respondan específicamente esta pregunta en términos de efectividad a largo plazo.

La hernia umbilical en pacientes asintomáticos con diástasis de los rectos asociada representa una condición que debe evaluarse dentro de un contexto estético y no funcional, siendo su tratamiento definitivo el resultado de una juiciosa evaluación de los riesgosbeneficios con el paciente, individualizando las expectativas y posibilidades para cada caso en particular.

\section{LA HERNIA UMBILICAL: UN EJEMPLO DE COMPLEJIDAD}

La cirugía de la hernia umbilical es considerada por los cirujanos, pacientes, administradores y aún en escenarios de aprendizaje de pre $y$ posgrado como un procedimiento sencillo, seguro, de bajo costo, fácilmente replicable y de alta efectividad clínica. No obstante, al profundizar en el tema vemos un abanico de posibles tratamientos, una incertidumbre en el logro de los mejores resultados para el paciente (impactos en las recidivas $\nabla \mathrm{s}$. impactos en las complicaciones) y pocos consensos entre los mismos cirujanos en los aspectos conceptuales y técnicos, constituyéndolo un escenario de complejidad dentro de lo aparentemente simple del tema ${ }^{(40)}$.

La evidencia disponible debe balancearse con la experiencia $y$ el juicio clínico del cirujano, tal y como se recomienda en el abordaje de los problemas complejos y no lineales, muy propios de los pacientes quirúrgicos. La solución debe plantearse de acuerdo con el paciente y su escenario, los recursos disponibles, las expectativas del paciente y la generación de consensos como herramienta fundamental en casos complejos, de acuerdo con lo expuesto por el profesor José Félix Patiño en su artículo sobre la cirugía y la evidencia ${ }^{(41)}$.
Por lo expuesto en el presente escrito, la hernia umbilical debe ser considerada, dentro de su sencillez, como un procedimiento desafiante, complejo y no debe ser minimizada.

\section{CONCLUSIONES}

La cirugía de la hernia umbilical es una intervención frecuente que exige del cirujano el conocimiento de las diferentes variables que inciden en la efectividad del procedimiento. EI uso de una prótesis genera mayor efectividad clínica con un incremento en los eventos adversos del sitio operatorio. La utilización de cirugía laparoscópica no confiere superioridad en la efectividad del procedimiento frente a la cirugía abierta, excepto en casos específicos. Es necesario dejar de considerar la hernia umbilical como un procedimiento simple, libre de complicaciones y relegada a los niveles más bajos del entrenamiento quirúroico.

\section{CONFLICTOS DE INTERESES}

Alejandro Lora y Neil Valentín Vega han trabajado para Johnson \& Johnson Medical S.A., en preceptoría y educación médica. Juan Pablo Ruiz ha trabajado para productos Winnermed S.A. en preceptoría y educación médica. Arnold José Barrios no declara conflictos de intereses. Todos los autores participaron en las diferentes fases de la elaboración del presente trabajo, no se recibieron fondos de alguna entidad estatal o privada y el mismo responde al esfuerzo y a los intereses académicos de sus autores.

\section{REFERENCIAS BIBLIOGRÁFICAS}

1. Kim LT. Complexity of the simple umbilical hernia repair. JAMA Surg. 2017;152(5):466. DOI 10.1001/jamasurg. 2016.5053.

2. Muysoms FE, Miserez M, Berrevoet F, Campanelli G, Champault GG, Chelala E, et al. Classification of primary and incisional abdominal wall hernias. Hernia. 2009;13(4):407-14. DOI 10.1007/s10029-009-0518-X.

3. Appleby PW, Martin TA, Hope WW. Umbilical Hernia Repair Overview of Approaches and Review of Literature. Surg Clin North Am. 2018;98(3):561-76. DOI 10.1016/j.suc.2018.02.001. 
4. Burcharth J, Pedersen MS, Pommergaard HC, Bisgaard T, Pedersen CB, Rosenberg J. The prevalence of umbilical and epigastric hernia repair: a nationwide epidemiologic study. Hernia. 2015;19(5):815-9. DOI 10.1007/s10029-015-1376-3.

5. Zens T, Nichol PF, Cartmill R, Kohler JE. Management of asymptomatic pediatric umbilical hernias: a systematic review. J Pediatr Surg. 2017;52(11):1723-31. DOI 10.1016/j.jpedsurg.2017.07.016.

6. Earle DB, McLellan JA. Repair of umbilical and epigastric hernias. Surg Clin North Am. 2013;93(5):105789. DOI 10.1016/j.suc.2013.06.017.

7. Toms AP, Dixon AK, Murphy JMP, Jamieson N V. Illustrated review of new imaging techniques in the diagnosis of abdominal wall hernias. $\mathrm{Br}$ J Surg. 1999;86(10):1243-9. DOI 10.1046/j.13652168.1999.01211.x.

8. Nguyen MT, Berger RL, Hicks SC, Davila JA, Li LT, Kao LS, et al. Comparison of outcomes of synthetic mesh $\nabla$ s suture repair of elective primary ventral herniorrhaphy: A systematic review and meta-analysis. JAMA Surg. 2014;149(5):415-21. DOI 10.1001/jamasurg. 2013.5014.

9. Winsnes A, Haapamäki MM, Gunnarsson U, Strigård K. Surgical outcome of mesh and suture repair in primary umbilical hernia: postoperative complications and recurrence. Hernia. 2016;20(4):509-16. DOI 10.1007/s10029-016-1466-X.

10. Shankar DA, Itani KMF, O’Brien WJ, Sanchez VM. Factors associated with long-term outcomes of umbilical hernia repair. JAMA Surg. 2017;152(5):461-6. DOI 10.1001/jamasurg.2016.5052.

11. Sanjay P, Reid TD, Daries EL, Arumugam PJ, Woodward A. Retrospective comparison of mesh and sutured repair for adult umbilical hernias. Hernia. 2005;9(3):248-51. DOI 10.1007/s10029-005-0342-X.

12. Kaufmann R, Halm JA, Eker HH, Klitsie PJ, Nieuwenhuizen J, van Geldere D, et al. Mesh versus suture repair of umbilical hernia in adults: a randomised, double-blind, controlled, multicentre trial. Lancet. 2018;391(10123):860-9. DOI 10.1016/S01406736(18)30298-8.

13. Bisgaard T, Kaufmann R, Christoffersen MW, Strandfelt P, Gluud LL. Lower Risk of Recurrence After Mesh Repair Versus Non-Mesh Sutured Repair in Open Umbilical Hernia Repair: A Systematic Review and Meta-Analysis of Randomized Controlled Trials. Scand J Surg. 2018. DOI 10.1177/1457496918812208.

14. Helgstrand F, Bisgaard T. Time for use of mesh repair for all umbilical hernias? Lancet. 2018;391(10123):821-2. DOI 10.1016/S0140-6736(18)30299-X.

15. Mirmehdi I, Ramshaw B. Synthetic Mesh: Making Educated Choices. In: Novitsky Yuri, editor. Hernia Surgery. Springer International Publishing Switzerland; 2016. p. 53-60.

16. Holihan JL, Nguyen DH, Nguyen MT, Mo J, Kao LS, Liang MK. Mesh Location in Open Ventral Hernia Repair: A Systematic Review and Network Metaanalysis. World J Surg. 2016;40(1):89-99. DOI 10.1007/ s00268-015-3252-9.

17. Tulloh B, de Beaux A. Defects and donuts: the importance of the mesh:defect area ratio. Hernia. 2016;20(6):893-5. DOI 10.1007/s10029-016-1524-4.

18. Ponten JEH, Leenders BJM, Leclercq WKG, Lettinga T, Heemskerk J, Konsten JLM, et al. Mesh Versus Patch Repair for Epigastric and Umbilical Hernia (MORPHEUS Trial); One-Year Results of a Randomized Controlled Trial. World J Surg. 2018;42(5):1312-20. DOI 10.1007/s00268-017-4297-8.

19. Berrevoet F, Doerhoff C, Muysoms F, Hopson S, Muzi MG, Nienhuijs S, et al. A multicenter prospective study of patients undergoing open ventral hernia repair with intraperitoneal positioning using the monofilament polyester composite ventral patch: Interim results of the PANACEA study. Med Devices (Auckl). 2017;10:81-8. DOI. 10.2147/MDER.S132755.

20. Hajibandeh S, Hajibandeh S, Sreh A, Khan A, Subar D, Jones L. Laparoscopic versus open umbilical or paraumbilical hernia repair: a systematic review and meta-analysis. Hernia. 2017;21(6):905-16. DOI 10.1007/s10029-017-1683-y.

21. Saber AA, Rao AJ, Itawi EA, Elgamal MH, Martinez RL. Occult ventral hernia defects: a common finding during laparoscopic ventral hernia repair. Am J Surg. 2008;195(4):471-3. DOI 10.1016/j.amjsurg.2007.04.013.

22. Bittner R, Bingener-Casey J, Dietz U, Fabian M, Ferzli GS, Fortelny RH, et al. Guidelines for laparoscopic treatment of ventral and incisional abdominal wall hernias (International Endohernia Society (IEHS) Part 1. Surg Endosc. 2014;28(1):2-29. DOI 10.1007/ s00464-013-3170-6. 
23. Lau H, Patil NG. Umbilical hernia in adults: Laparoscopic vs open repair. Surg Endosc. 2003;17(12):201620. DOI 10.1007/s00464-003-9027-7.

24. Warren JA, Cobb WS, Ewing JA, Carbonell AM. Standard laparoscopic versus robotic retromuscular ventral hernia repair. Surg Endosc. 2017;31(1):324-32. DOI 10.1007/s00464-016-4975-X.

25. Gonzalez A, Escobar E, Romero R, Walker G, Mejias J, GaIlas M, et al. Robotic-assisted ventral hernia repair: a multicenter evaluation of clinical outcomes. Surg Endosc. 2017;31(3):1342-49. DOI 10.1007/s00464-016-5118-0.

26. Kokotovic D, Sjølander H, Gögenur I, Helorstrand F. Watchful waiting as a treatment strategy for patients with a ventral hernia appears to be safe. Hernia. 2016;20(2):281-7. DOI 10.1007/s10029-016-1464-z.

27. Fueter T, Schäfer M, Fournier P, Bize P, Demartines N, Allemann P. The Hernia-Neck-Ratio (HNR), a Novel Predictive Factor for Complications of Umbilical Hernia. World J Surg. 2016;40(9):2084-90. DOI 10.1007/ s00268-016-3556-4.

28. Groene SA, Heniford DW, Prasad T, Lincourt AE, Augenstein VA. Identifying effectors of outcomes in patients with large umbilical hernias. Am Surg. 2016;82(7):613-21.

29. Strosberg DS, Pittman M, Mikami D. Umbilical hernias: the cost of waiting. Surg Endosc. 2017;31(2)9016. DOI 10.1007/s00464-016-5052-1.

30. Dokmak S, Aussilhou B, Belghiti J. Umbilical hernias and cirrhose. J Visc Surg. 2012;149(5)32-9. DOI 10.1016/j.jviscsurg.2012.04.002.

31. Saleh F, Okrainec A, Cleary SP, Jackson TD. Management of umbilical hernias in patients with ascites: Development of a nomogram to predict mortality. Am J Surg. 2015;209(2):302-7. DOI 10.1016/j.amjsurg. 2014.04.013.
32. Coelho JCU, Claus CMP, Campos ACL, Costa MA, Blum C. Umbilical hernia in patients with liver cirrhosis: A surgical challenge. World J Gastrointest Surg. 2016;8(7):476-82. DOI 10.4240/wjgs. $88 . i 7.476$.

33. Kulacoglu H. Umbilical Hernia Repair and Pregnancy: Before, during, after... Front Surg. 2018;5(1). DOI 10.3389/fsurg. 2018.00001 .

34. Haskins IN, Rosen MJ, Prabhu AS, Amdur RL, Rosenblatt S, Brody F. Umbilical hernia repair in pregnant patients: review of the American College of Surgeons National Surgical Quality Improvement Program. Hernia. 2017 Oct;21(5):767-770. DOI 10.1007/s10029-017-1633-8.

35. Oma E, Henriksen NA, Jensen KK. Ventral hernia and pregnancy: A systematic review. Am J Surg. 2018;217(1):1638. DOI 10.1016/j.amjsurg.2018.04.016.

36. Gilleard WL, Brown JM. The structure and function of the abdominal muscles during pregnancy and the immediate postbirth period. Phys Ther. 1992; 76(7):750-62. DOI 10.1093/ptj/76.7.750.

37. Juárez Muas DM. Preaponeurotic endoscopic repair (REPA) of diastasis recti associated or not to midline hernias. Surg Endosc. 2019 Jun;33(6):1777-82. DOI 10.1007/s00464-018-6450-3.

38. Naraynsingh $\nabla$, Maharaj R, Dan D, Hariharan S. Strong linea alba: Myth or reality? Medical hypotheses. Med Hypotheses. 2012;78(2):291-2. DOI 10.1016/j. mehy.2011.11.004.

39. Nahabedian MY. Management Strategies for Diastasis Recti. Semin Plast Surg. 2018;32(3):147-53. DOI 10.1055/s-0038-1661380.

40. PIsek PE, Greenhalgh T. Complexity science: The challenge of complexity in health care. Bmj. 2001;323(7313):625-8. DOI 0.1136/bmj.323.7313.625.

41. Patiño JF. ¿Existe la cirugía basada en la evidencia? Rev Colomb Cir. 2014;29:262-8. 